\title{
Groin and Medial Thigh Pain in a 17-year-old Girl
}

\author{
Lee J. Morse MD, Patrick P. Lin MD
}

Received: 8 June 2015/Accepted: 3 November 2015/Published online: 16 November 2015

(C) The Association of Bone and Joint Surgeons (R 2015

\section{History and Physical Examination}

An athletic and otherwise healthy 17-year-old girl presented to her pediatrician with a 2-year history of mild intermittent left groin and medial thigh pain of insidious onset and no history of trauma. She was 3-years postmenarche when her symptoms began. The patient described the pain as a "muscle-type ache" that occurred after playing soccer or cheerleading. Plain radiographs of the pelvis showed a bone lesion in the left inferior pubic ramus (Fig. 1A), after which a CT scan of the pelvis was obtained, and an orthopaedic oncology referral was made.

Each author certifies that he or she, or a member of his or her immediate family, has no funding or commercial associations (eg, consultancies, stock ownership, equity interest, patent/licensing arrangements, etc) that might pose a conflict of interest in connection with the submitted article.

All ICMJE Conflict of Interest Forms for authors and Clinical Orthopaedics and Related Research ${ }^{\mathbb{R}}$ editors and board members are on file with the publication and can be viewed on request.

Each author certifies that his or her institution approved the human protocol for this investigation, that all investigations were conducted in conformity with ethical principles of research, and that informed consent for participation in the study was obtained.

This work was performed at MD Anderson Cancer Center, Houston, TX, USA.

\section{J. Morse}

Department of Musculoskeletal Oncology and Orthopedic Surgery, Oakland Medical Center, Kaiser Permanente, Oakland, CA, USA

\section{P. P. Lin $(\square)$}

Department of Orthopaedic Oncology, MD Anderson Cancer

Center, 1515 Holcombe Boulevard, Unit 1448, Houston, TX

77030, USA

e-mail: plin@mdanderson.org
On presentation to our clinic, the patient reported no pain at the time of the visit, but rather she stated that the aching pain occurred only with activity, and generally persisted for 24 to 48 hours. She denied any fevers, chills, or other constitutional symptoms.

Physical examination showed a well-appearing 17-yearold adolescent girl in no apparent distress. She was afebrile and vital signs were within normal limits. She ambulated without a limp. Her examination was unremarkable except for mild pain in the region of the left ischial tuberosity with resisted knee flexion. There was no tenderness to deep palpation in that area. Otherwise she had full painless ROM of her hip and knee, with full strength and normal sensation throughout her left lower extremity. Serum laboratory tests were normal including the white blood cell count, erythrocyte sedimentation rate, and C-reactive protein.

\section{Imaging Interpretation}

A plain AP radiograph of the pelvis showed a well-marginated expansile lesion of the left inferior pubic ramus (Fig. 1). CT scans of the pelvis showed the presence of punctate calcifications within the lucent lesion and the cortices appeared more focally incomplete anteriorly and posteriorly (Fig. 2). Areas of endosteal scalloping also were appreciated. No soft tissue component was seen. MRI with gadolinium contrast revealed a lesion measuring 1.3 $\mathrm{cm} \times 1.3 \mathrm{~cm}$ (Fig. 3). The lesion had relatively low signal intensity on T1-weighted images but mirrored that of red marrow making it hyperintense to muscle (Fig. 3A). Intermediate signal intensity is seen on T2-weighted sequences (Fig. 3B) with peripheral contrast enhancement (Fig. 3C). No soft tissue component, periosteal reaction, or surrounding edema was appreciated. 

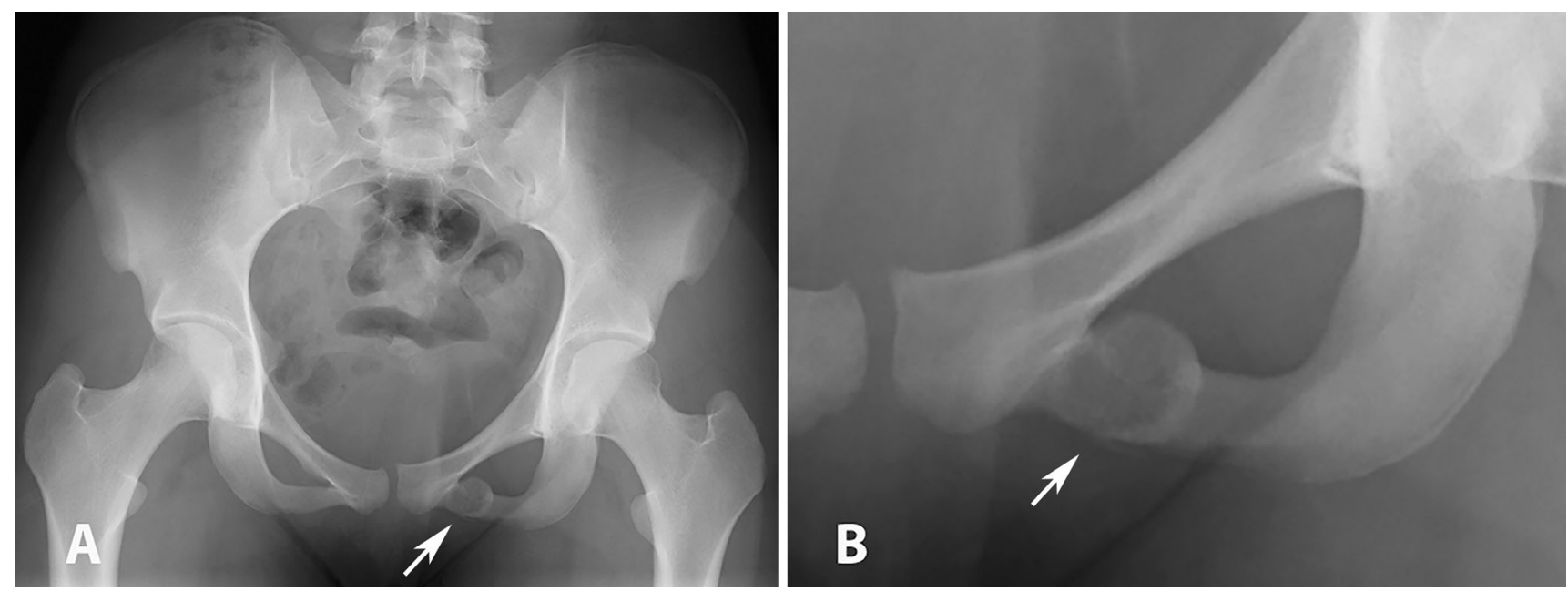

Fig. 1A-B (A) An AP pelvic radiograph and (B) an inset show a well-circumscribed expansile lucent lesion (arrow) in the left inferior pubic ramus.
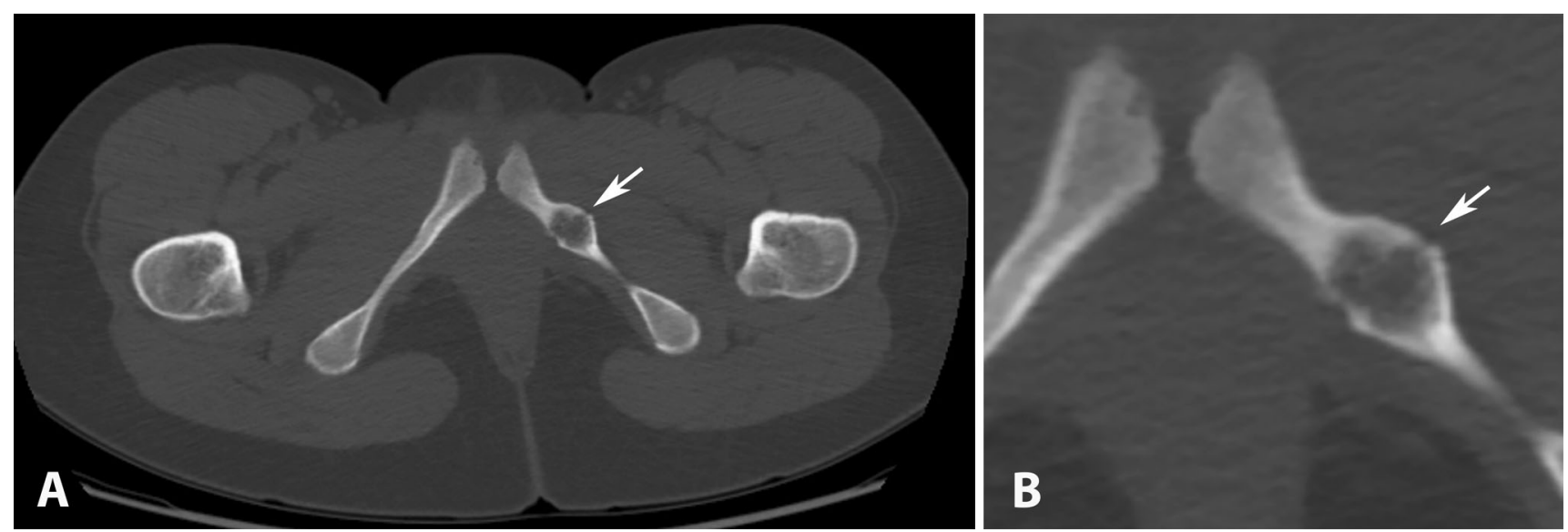

Fig. 2A-B (A) An axial CT scan of the pelvis and (B) an inset shows the lesion (arrow) with a sclerotic border and punctate calcifications. The cortices appeared more focally incomplete anteriorly and posteriorly.

Based on the history, physical examination, laboratory studies, and imaging studies, what is the differential diagnosis at this point?

\section{Differential Diagnosis}

Acute hematogenous osteomyelitis

Stress fracture

Enchondroma

Ewings sarcoma or osteosarcoma

Fibrous dysplasia

Eosinophilic granuloma

Bone cyst

Osteochondrosis

\section{Histology Interpretation}

An image-guided needle biopsy was performed and histologic analysis was done (Fig. 4). Grossly, the sample was an aggregate of pink bony tissue intermixed with coagulated blood. Histologically, the lesion contained sclerotic bony trabeculae (Fig. 4A) with remodeling in a background of fibrosclerotic and focal nonspecific fibrohistiocytic process (Fig. 4B). The paucicellular lesion contained rare multinucleated giant cells. Immunohistochemical stains for keratin and epithelial membrane antigen (EMA) were negative. CD68 staining highlighted the histiocytes.

Biopsy samples were sent for anaerobic, aerobic, fungal, and mycobacterial cultures. Corynebacterium minutissimum eventually grew, in broth medium only, of one specimen. 

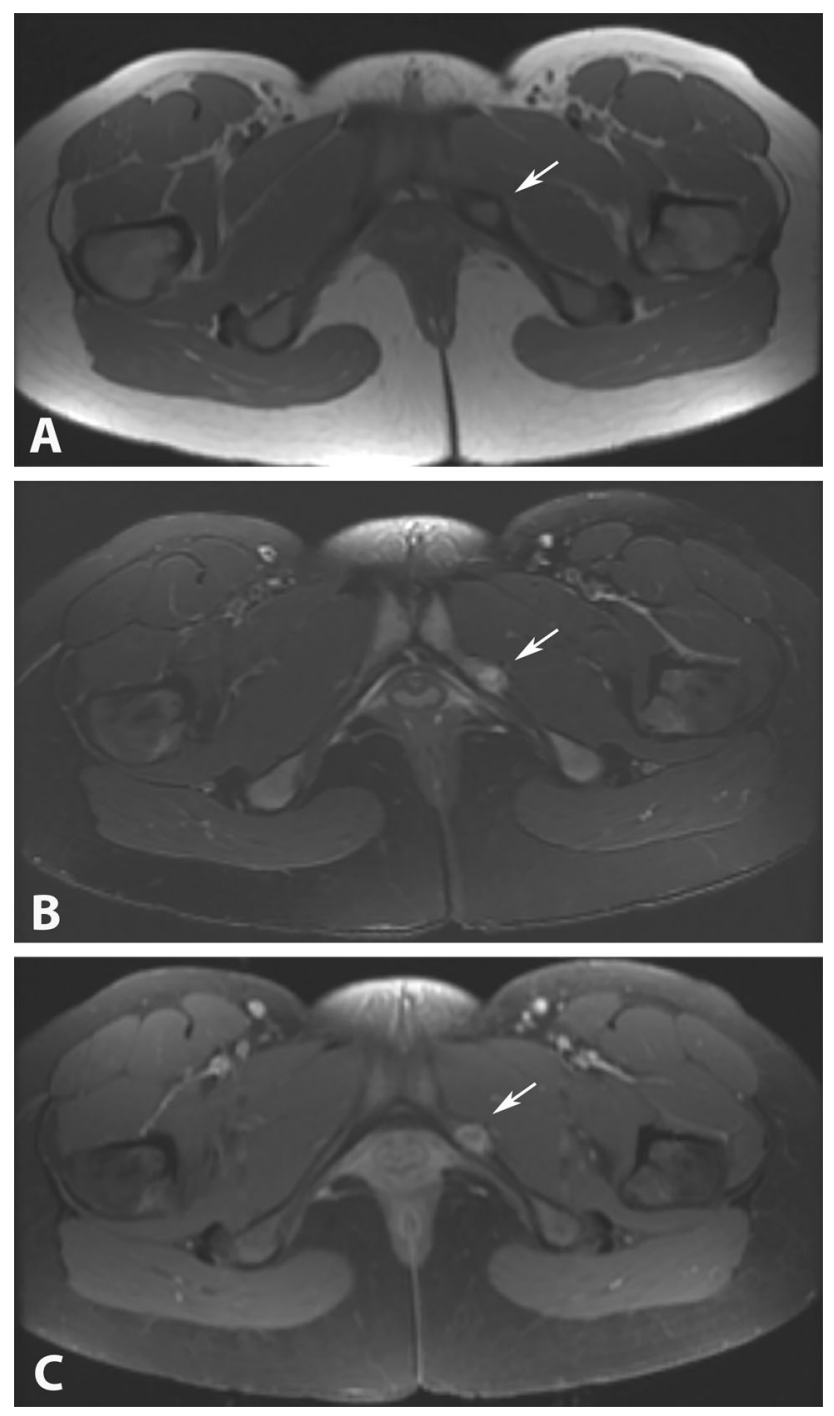

Fig. 3A-C Axial MR images of the pelvis show the lesion (arrow) has (A) low signal on T1-weighted images, but mirrors that of red marrow making it hyperintense to muscle, $(\mathbf{B})$ intermediate signal on T2-weighted images, and (C) enhances peripherally postgadolinium contrast. No soft tissue extension, marrow changes, or surrounding edema is seen.

Based on the history, physical examination, laboratory studies, imaging studies, and histology, what is the diagnosis and how should the patient be treated?

\section{Diagnosis}

Osteochondrosis of the ischiopubic synchondrosis (van Neck-Odelberg disease).

\section{Discussion and Treatment}

van Neck-Odelberg disease is a symptomatic enlargement of the ischiopubic synchondrosis and needs to be included in the differential diagnosis of a skeletally immature patient with groin or buttock pain. Patients often report mild groin or buttock pain that is exacerbated with activity, as was seen in our patient. Plain radiographic evaluation showed an expansile lucent lesion in the ischiopubic synchondrosis with well-defined borders but without periosteal reaction or significant bone destruction (Fig. 1), suggesting a benign etiology. MRI of the lesion showed low signal characteristics on $\mathrm{T} 1$ sequences but mirrored that of red marrow making it hyperintense to muscle (Fig. 3A), intermediate to high signal on T2 (Fig. 3B), and enhancement with gadolinium contrast (Fig. 3C). No soft tissue component, surrounding myositis, abscess, or free fluid was seen. The needle biopsy contained sclerotic bony trabeculae (Fig. 4 A) with remodeling in a background of fibrosclerotic and focal nonspecific fibrohistiocytic process (Fig. 4B). Byers [2] and Jarvis et al. [11], showed histologically, that osteochondroses are remnants of epiphyseal growth centers in van Neck-Odelberg disease with cortical bone and hyaline cartilage. The biopsy specimen from our patient did not contain hyaline cartilage, which could be attributable to sampling error or possibly the older age and skeletal maturity of the patient (17 years). Thus the histologic analysis in our patient did not confirm van NeckOdelberg disease, but rather was used to exclude other possibilities on our differential diagnosis list.

Although asymmetric enlargement of the ischiopubic synchondrosis is common, when symptomatic, the appearance of these "lesions" may force the clinician to consider infectious, neoplastic, or other etiologies leading to unnecessary tests and potentially morbid treatments. Our patient presented with an absence of fever, chills, or nonmechanical pain that would have been concerning for infection. Additionally, her erythrocyte sedimentation rate and C-reactive protein were within normal limits. On MR images, there was no enhancement of the surrounding soft tissues to suggest myositis, abscess, or free fluid, which also argues against infection. In general, osteomyelitis can be differentiated from van Neck-Odelberg disease by the absence of fever, chills, or nonmechanical pain; a negative erythrocyte sedimentation rate and C-reactive protein; and absence of surrounding soft tissue edema or abscess seen on MR images [13].

Stress fracture or avulsion injury could be considered in the radiographic and clinical differential diagnosis with the linear cortical disruption seen on CT scan (Fig. 2). However, the histologic analysis of our patient's biopsy specimen did not show any reparative process consistent with callus formation or bone healing. Punctate calcifications with endosteal scalloping were seen on CT scans, which could represent a cartilage neoplasm such as an enchondroma or a low-grade chondrosarcoma, but no chondroid cells were present in the biopsy specimen and 


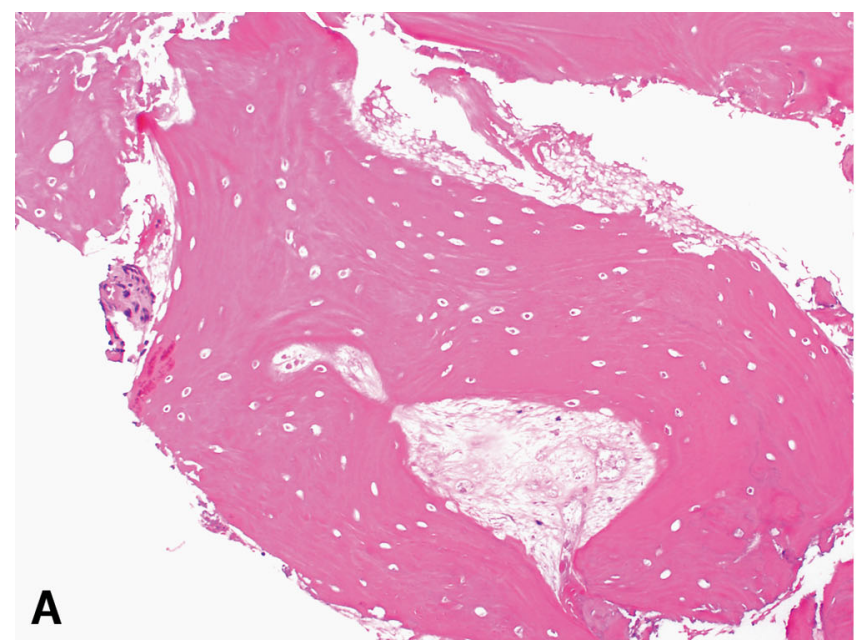

Fig. 4A-B (A) A representative slide from the biopsy specimen shows mature reactive bone formation is present (Stain, hematoxylin and eosin; original magnification, $\times 200)$. (B) There is sclerotic bony

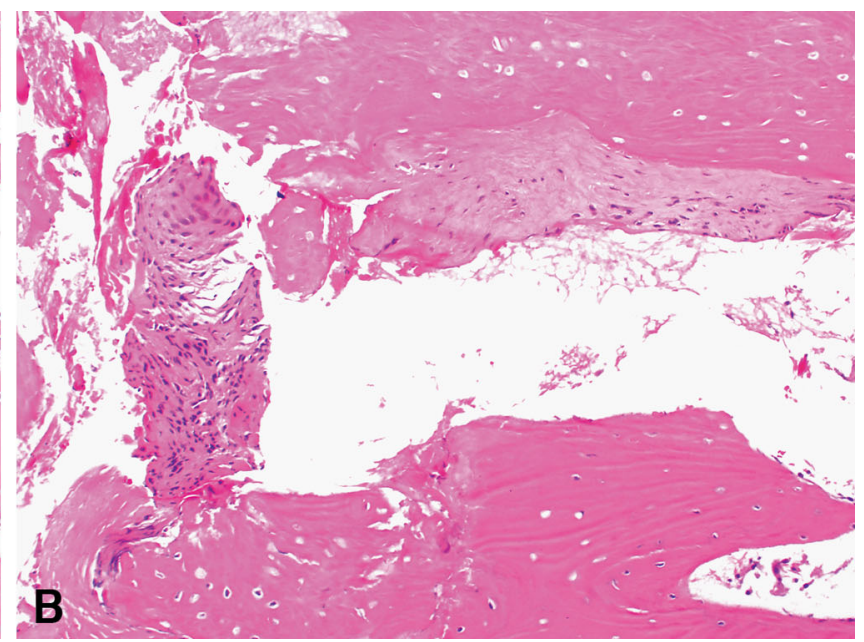

trabeculae with remodeling in a background of fibrosclerotic and focal nonspecific fibrohistiocytic process (Stain, hematoxylin and eosin; original magnification, $\times 200$ ).
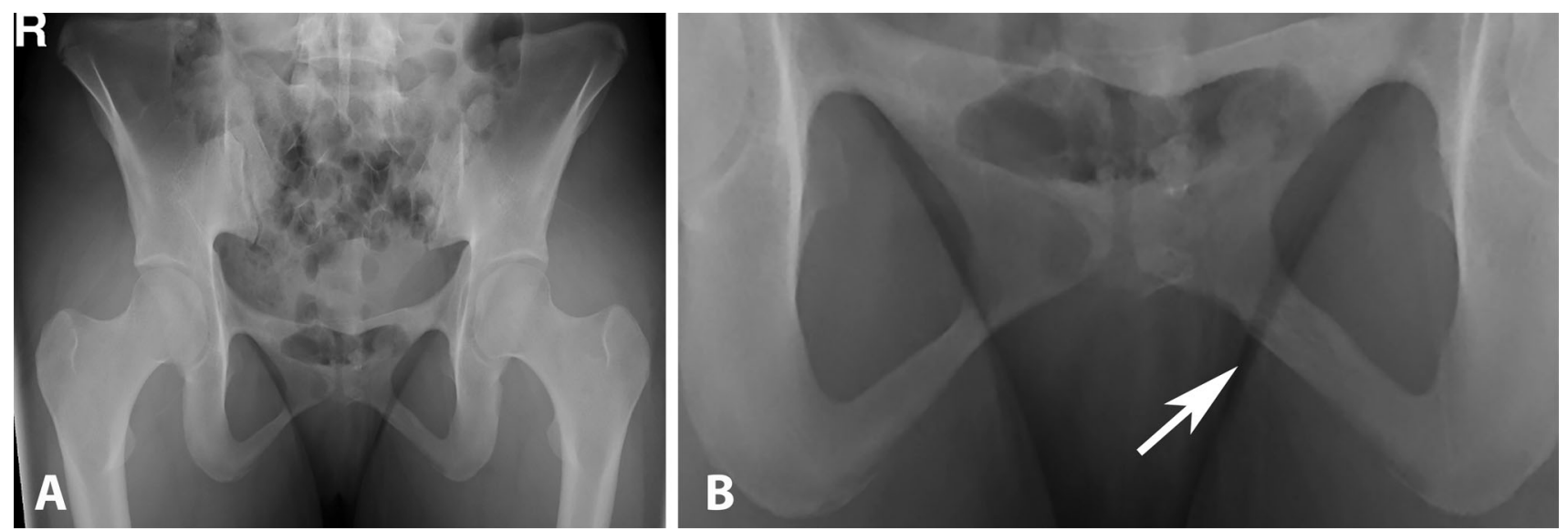

Fig. 5A-B (A) An AP pelvic radiograph and (B) an inset show near resolution of the left ischiopubic synchondrosis (arrow).

the patient's young age argues against a cartilage neoplasm. Furthermore, the histologic analysis did not show hypercellularity or any cellular atypia to suggest a malignant process such as Ewing's sarcoma or osteosarcoma. Although fibrous dysplasia can appear in any location and have similar radiographic characteristics as van NeckOdelberg disease, there were no representative histologic features consistent with fibrous dysplasia, such as fibroblastic proliferation surrounding woven bone.

Eosinophilic granuloma is considered a great mimicker on radiographs, and can occur in the pelvis; however, in our patient, the histologic features were paucicellular with only a few multinucleated giant cells and no histiocytelike cells or any cells with characteristic prominent nuclear grooves (coffee-bean nuclei). Although this lesion could represent an aneurysmal or unicameral bone cyst, the imaging and histologic analysis do not support either of those diagnoses. The lesion does not appear multiloculated, show any multifluid levels, nor appear overly aggressive like an aneurysmal bone cyst. No blood-filled caverns were seen on histologic analysis, and the rarity of giant cells also was not typical for aneurysmal bone cyst. The diagnosis of unicameral bone cyst likewise was not a good fit, either radiographically or histologically. The most common location for a unicameral bone cyst is the proximal humerus. Unicameral bone cysts are usually very dark on $\mathrm{T} 1$ images and very bright on $\mathrm{T} 2$ images, whereas our imaging showed low intensity on T1 but hyperintense to muscle and only intermediate signal on T2 images. No fibrous lining, no chronic inflammatory cells or other characteristics of unicameral bone cysts such as cementum-like spherules (calcified eosinophilic fibrinous material), or spindle cells were seen on histologic examination. 
The ischiopubic synchondrosis located at the junction of the inferior pubic ramus and ischial tuberosity behaves like a primary ossification center and fuses in late childhood to early adolescence. This normal fusion process may display bony enlargement, mineralization, and lucency, which are well described [1-6, 8-12]. Junge and Heuck [12] reported one in seven children 5 to 15 years old had "irregular" radiographic features of the ischiopubic synchondrosis in their series. These "irregular" features tend to be asymmetric [3, 5], and Caffey and Ross [3] reported a female predominance. Symptomatic enlargement of the ischiopubic synchondrosis initially was described by Odelberg [13] and van Neck [14] in 1923 and 1924, respectively. The five patients reported in these initial series showed nonspecific inflammatory changes on biopsy. All five patients were treated nonoperatively, and all symptoms eventually resolved. This condition has now been termed "osteochondrosis ischiopubica," "osteochondropathia ischiopubica," van Neck disease, or van Neck-Odelberg disease. Osteochondritis is a misnomer because there is no inflammatory process and osteochondrosis, although more appropriate, denotes skeletal abnormalities of the epiphysis or apophysis, not synchondroses. The etiology of this asymmetric expansion of the ischiopubic synchondrosis and why it becomes symptomatic in some patients has not been clearly answered. Some have theorized that a mechanical pull from the hamstrings causes micromotion at the ischiopubic synchondrosis causing formation of a callus leading to delayed ossification of the affected ischiopubic synchondrosis [7, 9]. Herneth et al. [9] theorized that van Neck-Odelberg disease arises more often in the nondominant lower extremity, as it did in our patient. Patients with these lesions should be followed with serial radiographs to ensure stability and no interval development of malignant features. To our knowledge, there are no large series with long-term followup data, but numerous investigators have suggested that van Neck-Odelberg disease should be treated nonoperatively with activity modification initially followed by progressive activity as symptoms resolve [2, 5, 12-15]. Although the radiologic findings tend to resolve with time, it actually is not known whether they completely disappear in all patients, and it is conceivable that subtle changes, such as slight expansion of bone, may persist on occasion.

As mentioned, our patient's clinical presentation was not consistent with an infectious etiology, and her imaging studies suggested a benign process. We initially recommended conservative management and close observation. We also advised activity modification with cessation of sports and impact exercises until symptom resolution. At her 4-month followup, there was a suggestion that the lesion had increased in size on repeat imaging studies. Because of this enlargement and because the patient was older than the typical patient with van Neck-Odelberg disease (younger than 12 years), a needle biopsy was performed, which confirmed a benign entity. Unexpectedly, cultures from the biopsy were positive for Corynebacterium minutissimum. This grew only in liquid medium, and thus, there was a strong possibility that it represented a contaminant from the skin. This bacterial species is associated with erythrasma, which is an asymptomatic skin rash found most commonly in the groin and axilla, resulting in dry skin with areas of pink or brown discoloration. Our patient had no such rash, but she was treated with 4 weeks of rifampin as a precaution based on the recommendation of the infectious disease specialist. At 6 months her symptoms were improving, and she was able to progress in her activities. At 1-year followup, the patient had returned to full sports and she was asymptomatic. The "lesion" had nearly disappeared radiographically (Fig. 5), in keeping with the diagnosis of van Neck-Odelberg disease.

Acknowledgments We thank Tamara Haygood MD (Department of Radiology, MD Anderson Cancer Center, Houston, TX), for radiographic assistance with diagnosis and Wei-Lien Wang MD (Department of Pathology, MD Anderson Cancer Center, Houston, $\mathrm{TX}$ ), for providing histologic images.

\section{References}

1. Angliss RD, Benson MK. Acetabular dysplasia and failure of development of the ischiopubic synchondrosis: a report of three related cases. J Pediatr Orthop B. 2000;9:248-251.

2. Byers PD. Ischio-pubic "osteochondritis": a report of a case and a review. J Bone Joint Surg Br. 1963;45:694-702.

3. Caffey J, Ross SE. The ischiopubic synchondrosis in healthy children: some normal roentgenologic findings. Am J Roentgenol Radium Ther Mucl Med. 1956;76:488-494.

4. Cawley KA, Dvorak AD, Wilmot MD. Normal anatomic variant: scintigraphy of the ischiopubic synchondrosis. J Nucl Med. $1983 ; 24: 14-16$.

5. Ceroni D, Mousny M, Anooshiravani-Dumont M, Buerge-Edwards A, Kaelin A. MRI abnormalities of the ischiopubic synchondrosis in children: a case report. Acta Orthop Belg. 2004;70:283-286.

6. Drubach LA, Voss SD, Kourmouzi V, Connolly LP. The ischiopubic synchondrosis: changing appearance on PET/CT as a mimic of disease. Clin Nucl Med. 2006;31:414-417.

7. Duben W. [Transformation zones in the infantile ischiopubic epiphysis and its significance in differential diagnosis] [undetermined language]. Chirurg. 1950;21:148-152.

8. Hardoff R, Gips S. Ischiopubic synchondrosis: normal finding, increased pubic uptake on bone scintigraphy. Clin Nucl Med. 1992;17:139.

9. Herneth AM, Philipp MO, Pretterklieber ML, Balassy C, Winkelbauer FW, Beaulieu CF. Asymmetric closure of ischiopubic synchondrosis in pediatric patients: correlation with foot dominance. AJR Am J Roentgenol. 2004;182:361-365.

10. Herneth AM, Trattnig S, Bader TR, Ba-Ssalamah A, Ponhold W, Wandl-Vergesslich K, Steinbach LS. MR imaging of the ischiopubic synchondrosis. Magn Reson Imaging. 2000;18:519524. 
11. Jarvis J, McIntyre W, Udjus K, Kloiber R. Osteomyelitis of the ischiopubic synchondrosis. J Pediatr Orthop. 1985;5: 163-166.

12. Junge H, Heuck F. [Osteochondritis ischipubica; simultaneous contribution to the normal development of the ischiopubic synchondrosis in growing children] [undetermined language]. Fortschr Geb Rontgenstr. 1953;78:656-668.
13. Odelberg A. Some cases of destruction in the ischium of doubtful etiology. Acta Chir Scand. 1923;56:273-284.

14. van Neck M. Osteochondrite du pubis. Arch Franco-Belges Chir. 1924;27:238-241.

15. Wait A, Gaskill T, Sarwar Z, Busch M. Van neck disease: osteochondrosis of the ischiopubic synchondrosis. J Pediatr Orthop. 2011;31:520-524. 